\title{
LA HISTORIA DE LA EDUCACIÓN MATEMÁTICA EN IBEROAMÉRICA
}

\section{The history of mathematics education in Latin America}

\author{
Dolores Carrillo Gallego, ${ }^{\alpha}$ José Manuel Matos, ${ }^{\beta}$ \\ Encarna Sánchez Jiménez y Wagner Rodrigues Valente ${ }^{\star}$
}

El «movimiento de historia de la educación matemática» ${ }^{1}$ que se ha ido desarrollando y diversificando a partir de la década de 1960, constituye actualmente una comunidad de investigación en auge.

Dentro de las sociedades científicas de Historia de la Matemática y de Educación Matemática (HME), se han constituido grupos de trabajo específicos sobre esta temática. Es el caso, en España, del grupo «Historia de las Matemáticas y Educación Matemática» (HMEM), dentro de la Sociedad Española de Investigación en Educación Matemática², y en Brasil del GHEMAT, «Grupo Associado de Estudos e Pesquisas sobre História

\footnotetext{
a Departamento de Didáctica de las Ciencias Matemáticas y Sociales. Facultad de Educación. Universidad de Murcia. Campus universitario de Espinardo, 30100 Murcia. España. carrillo@um.es (D) http://orcid.org/0000-0002-5170-2550

в Universidade Federal de Juiz de Fora e Universidade Nova de Lisboa. Instituto de Ciências Exatas - ICE. Rua José Lourenço Kelmer, s/n - Campus Universitário, Bairro São Pedro, 36036-900 Juiz de Fora, MG, Brasil. jmm@fct.unl.pt (iD https://orcid.org/0000-0003-2809-6561

> Departamento de Didáctica de las Ciencias Matemáticas y Sociales. Facultad de Educación. Universidad de Murcia. Campus universitario de Espinardo, 30100 Murcia. España. esanchez@um.es (D) https://orcid.org/0000-0001-5689-366X

* Departamento de Educação, Escola de Filosofia, Letras e Ciências Humanas, Universidade Federal de São Paulo - GHEMAT Brasil, Estrada do Caminho Velho, 333 - Jd. Nova Cidade - Guarulhos, SP CEP 07252-312. Brasil. ghemat.contato@gmail.com (1D http://orcid.org/0000-0002-2477-6677

${ }_{1}$ Wagner Rodrigues Valente, Introducción al no. 1 de la revista HISTEMAT. http://www.histemat. com.br/

${ }^{2}$ http://www.seiem.es/grp/hmem.shtml
}

Cómo citar este artículo: Carrillo Gallego, Dolores, José Manuel Matos, Encarna Sánchez Jiménez y Wagner Rodrigues Valente. "La historia de la educación matemática en Iberoamérica». Historia y Memoria de la Educación 11 (2020): 11-24 
da Educaçao Matemática » o el GHOEM, Grupo «História Oral e Educação Matemática», ${ }^{4}$ entre otros grupos; y en el ámbito internacional, el grupo HPE Grupo Internacional de Estudio sobre Relaciones entre Historia y Pedagogía de las Matemáticas, ${ }^{5}$ creado en 1980, vinculado a los congresos del International Congress of Mathematical Education (ICME) desde $1980^{\circ} \mathrm{o}$ la Comisión inter-IREM de Épistémologie et Histoire des Mathématiques, y los grupos de trabajo dentro de los Congresos de la Sociedad Europea de Investigación en Educación Matemática (CERME). Estos grupos convocan encuentros específicos dedicados a la HEM; así, en Brasil se realizan los ENAPHEM (Encontro Nacional de Pesquisas em História da Educação Matemática) y seminarios temáticos; en España, el grupo HMEM celebra reuniones intermedias, y a nivel internacional, el HPE se reúne cada cuatro años en los ICME Satellite Meetings of HPM, y organiza la «European Summer University on the History and Epistemology in Mathematics Education» con frecuencia trianual.

Los trabajos de estos grupos se publican en los Proceeding de las reuniones. ${ }^{7}$ Otros canales de difusión de los trabajos de Historia de la Educación Matemática son revistas tanto de Historia de las Matemáticas como de Educación Matemática o de Historia de la Educación, y es que, como recordaba Schubring en la presentación del primer número de la revista International Journal for the History of Mathematics Education, se trata de un campo interdisciplinar, en la confluencia de estos ámbitos de conocimiento; con ellos puede compartir cuestiones y métodos de investigación.

\footnotetext{
${ }^{3}$ https://www.ghemat-brasil.com/

${ }^{4}$ http://www2.fc.unesp.br/ghoem/

${ }^{5}$ https://www.mathunion.org/icmi/organizationaffiliated-organizations/hpm

6 Uffe Thomas Jankvist \& Jan van Maanen, «History and Mathematics Education», Recuperable en http://cerme10.org/wp-content/uploads/2017/01/TWG12_ERME_Book_Chp17_History_Draft.pdf

${ }^{7}$ Por ejemplo, Fulvia Furinghetti, Sten Kaijser, \& Constantinos Tzanakis (eds.), Proceedings of HPM 2004 \& ESU 4: ICME 10 Satellite Meeting of the HPM Group \& Fourth European Summer University on the History and Epistemology in Mathematics Education (Iraklion, Greece: University of Crete, 2006); Marta Pytlak, Tim Rowland y Ewa Swoboda (eds.). Proceedings of the Seventh Congress of the European Society for Research in Mathematics Education (Rzeszów: University of Rzeszów, 2011), 1636-1639.; Behiye Ubuz, Cigdem Haser y Maria Alessandra Mariotti (eds.), Proceedings of the Eighth Congress of the European Society for Research in Mathematics Education (Ankara: METU, 2013), 1945-1950.; Y Nada Vondrová y Konrad Krainer (eds.), Proceedings of the Ninth Congress of the European Society for Research in Mathematics Education (Prague: Charles University, 2015), 1783-1789.
} 
Otra forma de difusión de estos resultados son las obras específicas, como el NCTM Yearbook de 1969,8 la obra de Karp y Schubring, ${ }^{9}$ o los libros publicados por el GHEMAT que recogen sus investigaciones ${ }^{10}$. También se han utilizado revistas específicas, como la ya citada International Journal for the History of Mathematics Education, publicada entre 2006 y 2016. En la actualidad, en Brasil se publica HISTEMAT, ${ }^{11}$ que se presenta como «veículo representativo do movimento da história da educação matemática», y «lugar de sistematização dos avanços da história da educação matemática como área científica». ${ }^{12}$

\footnotetext{
${ }_{8}$ NCTM Yearbook, Historical topics for the mathematics classroom (Reston, Virginia: NCTM, 1969). Updated edition 1989.

9 Alexander Karp \& Gert Schubring (eds.), Handbook on the History of Mathematics Education (New York: Springer, 2014).

10 Wagner Rodrigues Valente, A matemática na formação do professor do ensino primário. São Paulo, 1875-1930 (Sao Paulo: GHEMAT, 2011); Wagner Rodrigues Valente (org.), Avaliação em matemática: história e perspectivas atuais. (Sao Paulo: GHEMAT, 2012); José Manuel Matos y Wagner Rodrigues Valente (orgs.), A Matemática Moderna em contextos Ibero-Americanos (Lisboa, 2010). http://run.unl.pt/bitstream/10362/5321/1/Matos_2010.pdf. Maria Cristina Araújo de Oliveira, Maria Célia Leme da Silva y Wagner Rodrigues Valente (orgs.), O movimento da Matemática Moderna: história de uma revolução curricular (Sao Paulo: GHEMAT, 2011); Cláudia Flores y Joseane Pinto de Arruda (orgs.), A Matemática Moderna nas escolas do Brasil e Portugal. Contribuição para a história da educação matemática (Sao Paulo: GHEMAT, 2010); Maria Célia Leme da Silva y Wagner Rodrigues Valente (orgs.), Na oficina do historiador da educação matemática. Cadernos de alunos como fonte de pesquisa (Sao Paulo: GHEMAT, 2010); Wagner Rodrigues Valente (org.), Osvaldo Sangiorgi - um professor moderno (Sao Paulo: GHEMAT, 2008); Elisabete Zardo Búrigo, Maria Cecília Bueno Fischer y Monica Bertoni dos Santos (orgs.), A Matemática Moderna nas escolas do Brasil e de Portugal: novos estudos (Sao Paulo: GHEMAT, 2009); Wagner Rodrigues Valente (org.), A Matemática moderna nas escolas do Brasil e de Portugal: Primeiros estudos (Sao Paulo: GHEMA, 2007); Wagner Rodrigues Valente (org.), Ubiratan D'Ambrosio (Sao Paulo: GHEMAT, 2007); Wagner Rodrigues Valente (org.), O Nascimento da Matématica do Ginásio (Sao Paulo: GHEMAT, 2003); Wagner Rodrigues Valente, Uma História da Matemática Escolar no Brasil (1730-1930) (Sao Paulo: GHEMAT, 1999); Wagner Rodrigues Valente (org.), Euclides Roxo e a modernização do ensino da matemática no Brasil (Sao Paulo: GHEMAT, 2004); David Antonio da Costa y Wagner Rodrigues Valente (orgs.), Saberes matemáticos no curso primário: o que, como e por que ensinar? (São Paulo: GHEMAT, 2014); Neuza Bertoni Pinto y Wagner Rodrigues Valente (orgs.), Saberes elementares matemáticos em circulação no Brasil: dos documentos oficiais às revistas pedagógicas, 1890-1970 (São Paulo: GHEMAT, 2016); Iran Abreu Mendes y Wagner Rodrigues Valente (orgs.), A matemática dos manuais escolares - curso primário, 1890-1970 (São Paulo: GHEMAT, 2017); Renaud D’Enfert, Marc Moyon y Wagner Rodrigues Valente (orgs.), Les mathématiques à l'école élémentaire (1880-1970). Études France-Brésil (Limoges: GHEMAT, 2017); Diogo Rios, Elisabete Zardo Búrigo, Maria Cecília Fischer y Wagner Rodrigues Valente (orgs.), Cadernos escolares e a escrita da história da educação matemática (São Paulo: GHEMAT, 2017). E Ivanildo Lima, Luciane Bertini, Maria Cristina Araújo de Oliveira, Elisabete Zardo Búrigo y Wagner Rodrigues Valente (orgs.), Provas, Exames e História da Educação Matemática (Roraima: GHEMAT, 2018).

${ }^{11} \mathrm{http}: / /$ www.histemat.com.br/

12 Presentación del no. 1 de HISTEMAT.
} 
Una de las riquezas del ámbito de investigación de la HEM lo constituye la diversidad de enfoques relacionados con los distintos intereses de los investigadores y las influencias que reciben. Además, se considera que la HEM aporta conocimientos útiles tanto a los investigadores en Educación Matemática, pues el análisis de los conceptos matemáticos investigados se puede enriquecer al considerar su desarrollo histórico, como a los profesores de matemáticas, en especial a los dedicados a la formación del profesorado.

Con motivo del 20 aniversario, en 2017, de la Sociedad Europea de Educación Matemática (ERME), en cada uno de los grupos de trabajo del CERME 10 se presentó un documento para la discusión. En el grupo TW12 «History in Mathematics Education», Uffe Thomas Jankvist y Jan van Maanen presentaron el documento titulado «History and Mathematics Education», en el que identifican dos líneas de trabajo distintas en este grupo que considera la educación matemática desde una perspectiva histórica:

a) Historia de la Matemática en la Educación Matemática, que es la línea que se refleja en la denominación del grupo.

b) Historia de la Educación Matemática, presente, de forma creciente, a partir del CERME $6 .{ }^{13}$

Estas dos orientaciones se manifiestan también en la existencia de dos Topics Study Groups (TSG) en los ICME; en el ICME 2020, que se va a celebrar en Shangai, el TSG27 se dedicará a «The role of the history of mathematics in mathematics education», mientras que el TSG55, "The history of the teaching and the learning of mathematics» estará coordinado por el profesor Wagner Rodrigues Valente.

Los Congresos Iberoamericanos de Historia de la Educación Matemática (CIHEM) «complementa e aprofunda o intercâmbio entre pesquisadores e a produção de conhecimento ligada à História da Educação Matemática na América Latina, em Portugal e Espanha, espelhando as diversas perspectivas e metodologias que têm vindo a ser seguidas».${ }^{14} \mathrm{Se}$

\footnotetext{
13 Jankvist van Maanen, «History and Mathematics Education».

14 Miguel Chaquiam, Iran Abreu Mendes y Wagner Rodrigues Valente, III Congresso Ibero-Americano História da Educação Matemática. Anais (Belem, Brasil, 2015), 9. https://repositorio.ufsc.br/xmlui/handle/123456789/169988.
} 
han realizado cinco ediciones de estos congresos, celebradas en Covilhã (Portugal, 2011), Cancún (México, 2013), Belem do Pará (Brasil, 2015), Murcia (España, 2017) y Bogotá (Colombia, 2019).

El IV CIHEM fue organizado por el Centro de Estudios sobre la Memoria Educativa (CEME) de la Universidad de Murcia, y sus objetivos fueron:

- Profundizar en el intercambio entre investigadores de la historia de la educación matemática en América Latina, en Portugal y en España.

- Dar a conocer las diversas perspectivas, temas y metodologías seguidas hasta el momento.

- Facilitar la producción y difusión de conocimiento en este ámbito.

- Promover la formación de grupos de trabajo y la colaboración entre los ya existentes.

- Analizar el estado actual de este campo de investigación en auge y sus perspectivas de futuro. ${ }^{15}$

Este monográfico recoge una muestra de trabajos de los investigadores que intervinieron en dicho IV CIHEM.

La profesora Maria Cristina Araújo de Oliveira, en su trabajo «História da educação matemática, saberes objetivados e a constituição de uma disciplina», explora el interés que puede tener el conocimiento de la HEM para los profesores de matemáticas y, en relación con ese interés, la inclusión de la HEM en la formación inicial del profesorado y el estado de la formación, en Brasil, de una disciplina, la HEM. Utilizando un trabajo de 2019 del profesor Valente, estudia la noción de «saber objetivado» y su relación con los saberes profesionales y, en particular, con la fomación de profesores. La autora considera que «uma das finalidades da História da educação matemática enquanto campo de investigação, produzir trabalhos ou criar meios que permitam aos futuros professores conhecer e se apropriar da matemática escolar numa perspectiva histórica». Comenta el modelo de desarrollo de una materia escolar propuesto por Goodson

\footnotetext{
15 https://www.um.es/cihem4/
} 
y considera que, en el caso de la disciplina de HEM se relaciona con la dimensión profesional de la formación de los profesores de matemáticas. También presenta los estudios de Hofstetter y Schneuwly sobre el proceso de disciplinarización y lo compara com la situación de la comunidad de investigadores en el área de HEM en Brasil. Por último, tras comentar algunos trabajos sobre la evolución del currículo de matemáticas en Brasil, se presentan las experiencias de introducción de la asignatura de HEM en licenciaturas y posgrados, que se están llevando a cabo en diversas universidades brasileñas, comparando orientaciones, temarios y bibliografía. Señala que la HEM también influye en la docencia en otras asignaturas cuando son impartidas por investigadores de HEM. Se destaca que la orientación dada a la asignatura en las distintas universidades depende de las personas que son sus responsables. Esta variabilidad local caracteriza la situación actual de la constitución de la HEM como disciplina en Brasil.

José María Muñoz-Escolano y Antonio M. Oller Marcén utilizan una fuente poco usada en historia de la educación matemática: los prólogos de los libros de texto, en particular, de los textos algebraicos publicados en España en el siglo XVI. Esta fuente, incluida en el grupo de las consideradas "paratextos», aporta información valiosa no solo sobre aspectos más genéricos, como las intenciones del autor o autores, o lo novedoso que pueda resultar un manual en comparación con los existentes hasta el momento; los prólogos permiten conocer, además, cómo conciben los autores la matemática y sus ideas acerca de cómo se aprende y cómo debe ser enseñada. En su artículo recogen varios descriptores con relación a los cuales estudiar los prólogos, como el tipo de prólogo, la función que le asigna su autor, así como unas categorías para el análisis de concepciones y creencias, y una clasificación de éstas. Más allá de la información que aportan sobre unos libros de texto concretos, la lectura de este trabajo es útil al investigador en HEM por su aportación al conocimiento sobre el papel de los prólogos para el estudio histórico de los libros de texto de matemáticas.

El trabajo de Luis Carlos Arboleda aborda una cuestión importante para la matemática: el paso de un enfoque sintético a otro analítico. Lo hace a través de la trayectoria del profesor José Celestino Mutis y su evolución al frente de las enseñanzas de matemáticas en el Colegio del Rosario, un centro significativo de Colombia. Partiendo de una caracterización del método de análisis-síntesis a lo largo de la historia, el autor 
destaca lo que supuso la transformación de la matemática entre los siglos XVIII y XIX. Hace un recorrido por el pensamiento y la trayectoria de Mutis y la orientación que imprimió a las matemáticas en la cátedra de la que era responsable, desde un enfoque basado en la obra de Wolf, pasando por su interpretación de la obra de Descartes y su evolución desde un enfoque logicista hasta la institucionalización de la visión newtoniana. En este recorrido incluye las influencias gaditanas que recibió Mutis para escribir sus Elementos en 1764, hasta la de Bails, cuya obra introduce en Colombia, buscando difundir una concepción más moderna de las matemáticas, a la vez que de su enseñanza.

Por su parte, Isabel Sánchez y María Teresa González eligen un militar español que desarrolló su labor docente y sus publicaciones en el siglo XIX, José Odriozola, para analizar el modo en el que propone enseñar la geometría analítica en las academias militares, aunque el Curso Completo de Matemáticas Puras fuera recomendado también para la segunda enseñanza. Las autoras, tras algunos datos biográficos, comparan la obra de Odriozola con la de otros autores contemporáneos y estudian lo que supuso la obra de este autor, en particular su relación con el trabajo de Descartes. Para ello caracterizan las dos formas de abordar la geometría analítica, o de aplicar el álgebra a los problemas geométricos, en el libro de Odriozola: una más próxima al enfoque de Descartes, y otra que emplea los sistemas de coordenadas e intenta superar las limitaciones del tratamiento anterior. Ante todo, basándose en los ejemplos analizados y, a la vez, en los comentarios y explicaciones que revelan las ideas de este profesor acerca de estas cuestiones, en el trabajo se estudia la manera en la que se resuelven los problemas en el manual de Odriozola y la relación que se establece entre geometría y álgebra, característica de este autor y una aportación singular en aquel momento en el contexto español.

La contribución de Miguel Picado y Jonathan Espinoza presenta varias obras de aritmética para la formación de los maestros costarricenses en el siglo XIX, y analiza en detalle una en particular, editada en 1897, el Manual para el maestro. Curso elemental de aritmética, arreglado de acuerdo con los programas oficiales, de Félix Francisco Noriega. Comienzan ofreciendo una breve panorámica de la formación de los maestros en ese país, en las Secciones Normales de los Institutos de secundaria, antes de la creación de la Escuela Normal, ya en 1914, y la distancia 
que mantiene aquella formación con una verdadera profesionalización del magisterio primario. Continúan describiendo los contenidos de las obras de aritmética de Osejo y de Salazar, publicada la primera en el primer tercio de siglo y la segunda una década antes de la publicación del libro de Noriega, que los autores del artículo analizan, tras algunas consideraciones metodológicas. Utilizan para el análisis distintas categorías pertenecientes a dos niveles, categorías del análisis didáctico sobre lo cognitivo, de instrucción y de evaluación y, además, otras secundarias relativas a los conceptos y procedimientos aritméticos asociados a las sugerencias didácticas para la enseñanza que contiene el manual. Este análisis comprende aspectos diversos, como las tareas propuestas, el papel y uso de los materiales y otros recursos, la actuación ante las dificultades de los niños o aspectos relativos a la manera en la que el profesor debe abordar los contenidos aritméticos y el papel de la evaluación, entre otros. Finalmente, el estudio realizado aporta una mejor comprensión de la formación matemática pretendida para los maestros en esos momentos en Costa Rica.

Mária Cristina Almeida aborda el tema de la elaboración y la selección de los libros de texto, y lo hace a partir del análisis de un manual para el tercer curso del liceo en Portugal, el Compêndio de Álgebra de António Augusto Lopes, publicado en 1950 y usado durante el Estado Nuevo (1947-1974), en el que se instauró el «libro único». En este caso se trata de comprender mejor cómo se enseñaba el análisis infinitesimal en el nivel de enseñanza mencionado, además de la polémica generada en torno a la publicación de esta obra. Para ello, la autora del trabajo comenta las críticas de algunos matemáticos portugueses, vertidas en los artículos publicados en dos revistas, Labor y Gazeta de Matemática, así como la respuesta del propio Lopes, y compara la primera edición del libro con otra posterior. A partir de las críticas surgidas con motivo de la adopción de la obra como libro de texto para los liceos, la autora del trabajo da a conocer la preocupación en aquel momento por varias cuestiones en relación con la enseñanza del cálculo infinitesimal, que se refieren principalmente a la dialéctica entre intuición y rigor y al papel del análisis infinitesimal en ese nivel de enseñanza. En el artículo se analizan las observaciones relativas a los errores o la falta de precisión cometidos en algunas definiciones fundamentales, como las de límite o continuidad, así como la falta de rigor en el lenguaje matemático y en 
algunas demostraciones y, sobre todo, al modo de presentar la materia a los adolescentes. Tras esto se compara la edición primera con otra posterior, prestando atención a cada una de las críticas recibidas a propósito del primer libro, y se constata, como reconoce el propio autor, cómo toma en consideración la mayoría de los puntos que se le señalan, y a qué tipo de modificaciones dan lugar en la edición posterior, con especial atención a aquellos que dejan traslucir motivaciones didácticas, los relativos a lo que Shulman denomina conocimiento pedagógico del contenido.

La profesora Rosilda Morais comienza aclarando la distinción entre «Resolução de Problemas» y «resolução de problemas» y caracterizando esta última como un constructo que engloba al anterior pero no se reduce a él. Se pregunta entonces por la manera de fomentar en el aula una cultura de resolución de problemas y la competencia necesaria en el profesor para ello. El trabajo se centra en las aportaciones de George Polya, evitando la habitual perspectiva reduccionista en torno a la obra de Polya, centrada en las cuatro fases que describe. Al contrario, considera la obra de Polya en tanto que este se interesa por la enseñanza «sobre resolución de problemas», es decir, que su objetivo cuando trató la resolución de problemas no era otro que la formación de profesores de matemáticas. Esta autora, siguiendo sobre todo a Hofstetter y Schneuwly, sitúa los saberes en el centro de las instituciones de enseñanza y de formación, y considera tanto los saberes a enseñar como los saberes para enseñar constitutivos del campo profesional. Frente a la consideración de este tópico como recurso para la enseñanza, Morais aboga por problematizar la resolución de problemas como un conocimiento, esto es, como parte de la matemática para enseñar. Para la autora, Polya precisamente reivindicaba para la resolución de problemas un estatus epistemológico en tanto que saber para enseñar, es decir, como un saber propio de la formación de profesores, o saber profesional.

El monográfico se completa con cuatro trabajos con valor documental.

El estudio Patrimonio Iberoamericano en Matemáticas y en Educación Matemática (PIMEM), que presenta el profesor Luis Rico, parte de la invitación por parte de la International Commission for Mathematical Instruction (ICMI) a la Comisión de Educación del Comité Español de Matemática, para que contribuyera con una exposición y una conferencia 
durante el ICME de 2012. Invitación que dio lugar a la exposición Spanish Heritage in Mathematics and Mathematics Education y a la conferencia, de igual título, pronunciada por quien en aquellos momentos era el presidente de la Comisión de Educación del CEMat, y autor del trabajo que aparece en este monográfico. Expertos en historia de la matemática y de la educación matemática de varias universidades e instituciones iberoamericanas elaboraron los pósteres que componen la exposición, que ofrece una síntesis diacrónica de la historia científica y matemática, compartida por España y el resto de países de América. Esta exposición, ampliada posteriormente hasta los 40 pósteres y traducida al español, participó en numerosos congresos y encuentros en varios países y, finalmente, en el IV CIHEM. El profesor Rico en su informe destaca el papel de algunas instituciones, como la Organización de Estados Iberoamericanos para la Educación, la Ciencia y la Cutura (OEI), organismo intergubernamental de la Comunidad Iberoamericana de Naciones (CNI), y de otras instituciones participantes, y los criterios con los que se elaboraron los pósteres. La exposición se estructura en cinco periodos, en torno a los cuales se sitúan los temas tratados (siglos XV y XVI, siglos XVII y XVIII, siglo XIX, siglo XX y, por ultimo, siglo XXI). En este trabajo se enumeran los temas elegidos en cada uno de los periodos y se resume brevemente su contenido destacando a la vez los hechos cruciales en cada uno de ellos, tanto por lo que respecta a la matemática como a su enseñanza, y los personajes protagonistas de algunos de ellos. Ante todo, representa una oportunidad para, en palabras de Rico, «contraponer o integrar pensamientos y juicios surgidos de lecturas e interpretaciones coherentes acerca de la matemática y la educación matemática como parte esencial de nuestra lengua y nuestra cultura».

Pilar Orús y Dilma Fregona nos ofrecen una panorámica retrospectiva de lo que ha supuesto en didáctica de la matemática la conocida como "corriente francesa», surgida en la década de los setenta del siglo pasado y, muy especialmente, la figura de Guy Brousseau, para el nacimiento de un paradigma de investigación en didáctica de la matemática, que ha «emancipado» a esta disciplina de las ciencias de la educación, en el sentido de imprimirle un carácter propio, otorgando al saber matemático un papel fundamental. Tras una breve reseña sobre la historia de la educación matemática en Francia y algunas instituciones relacionadas, 
las autoras describen la Teoría de las Situaciones Didácticas (TSD), centrándose en su nacimiento y destacando el papel no solo de las personas, sino de las instituciones que favorecieron su desarrollo y de las iniciativas, publicaciones y eventos asociados. En especial son de agradecer sus referencias sobre el Grupo Escolar Jules Michelet y el Centre d'Observation et de Recherche pour l'Enseignement des Mathématiques (COREM), cómo este centro permitió llevar a cabo una metodología basada en la "Observación», y la descripción de los instrumentos metodológicos asociados a ella y vinculados a la escuela Michelet, la relación del Centro con los Instituts de Recherche pour l'Enseignement des Mathématiques (IREM), la Association pour la Recherche en Didactique des Mathématiques (ARDM), etcétera. Estas instituciones desempeñaron un papel fundamental en las investigaciones llevadas a cabo en Francia y en el nacimiento de la llamada «didáctica fundamental». Esta panorámica que nos presentan sobre las instituciones francesas abarca un breve recorrido por la difusión del citado paradigma investigador en Didáctica de la Matemática en España, que daría lugar al nacimiento del Seminario Interuniversitario de Investigación en Didáctica de las Matemáticas (SIIDM) en 1991, integrado después en la Sociedad Española de Investigación en Educación Matemática (SEIEM). E igualmente la creación de un campo de investigación sobre la educación matemática en Argentina, desde las actividades vinculadas a la Reunión de Educación Matemática (REM) hasta la creación de la Sociedad Argentina de Educación Matemática (SOAREM), y las relaciones entre la comunidad investigadora argentina e investigadores franceses como Michèle Artigue, Yves Chevallard o el propio Brousseau. La última parte del trabajo se dedica a los recursos producidos por el COREM, y a la creación en la Universidad Jaime I de Castellón de la Plana (España), del Centro de Recursos de Didáctica de las Matemáticas CRDM-Guy Brousseau, que alberga la colección de materiales en soporte papel del que fuera el COREM durante su existencia entre 1972 y 1999. Acaba con un ejemplo de una investigación realizada a partir de los recursos del CRDM que trata de ilustrar, por un lado, la manera de localizar y consultar el material disponible y, por otro, el potencial que supone este para investigaciones actuales.

El profesor Wagner Rodrigues Valente presenta un grupo constituido por investigadores pertenecientes a universidades de todo Brasil, reunidos 
en torno a proyectos de investigación sobre la historia de la educación matemática en ese país. Comienza con una breve referencia al nacimiento de la comunidad de investigadores brasileños en educación matemática, con la creación en 1988 de la Sociedade Brasileira de Educação Matemática, reuniones como Encontros Nacionais de História da Educação Matemática (ENAPHEM), y eventos internacionales, como los Congresos Iberoamericanos de Historia de la Educación Matemática (CIHEM), además de publicaciones, entre las que destaca HISTEMAT (Revista de História da Educação Matemática). El trabajo presenta la trayectoria seguida en este país desde la creación en el año 2000 del grupo GHEMAT-SP hasta la reciente constitución de GHEMAT Brasil (Grupo Associado de Estudos e Pesquisas sobre História da Educação Matemática), resultado de una labor comenzada en São Paulo, y del trabajo realizado por un grupo cada vez más amplio de investigadores, no solo paulistas, que buscaron y consiguieron trabajar conjuntamente con Portugal y Francia en grupos de investigación internacionales en los que participan profesores de varias universidades de los países implicados. Entre los fines del grupo están la promoción y la divulgación de estudios sobre historia de la educación matemática, a través de seminarios, congresos y reuniones, la formación de investigadores, las publicaciones y el establecimiento de convenios con instituciones que compartan el interés por la investigación en este campo disciplinar. Una de las señas de identidad del grupo es la manera en la que entiende la forma de hacer investigación, partiendo de la Historia de la Educación y teniendo como referencia la formación en matemáticas y en educación matemática. Durante casi una década el interés se centró en la constitución de una disciplina en torno a la matemática para enseñar en la etapa secundaria —nivel en el que la enseñanza estuvo en Brasil centralizada, al contrario de lo que sucedió con la etapa primaria—, así como en la caracterización del saber profesional del profesor de matemáticas.

Por último, el artículo describe uno de los principales logros del GHEMAT, la creación del Centro de Documentación del GEHMAT-SP, que contiene no solo obras didácticas de matemáticas sino también archivos personales de personajes icónicos de la educación matemática brasileña, algunos tan relevantes como Euclides Roxo y otros más actuales, entre los que podemos citar a Urbiratán D'Ambrosio. Pero la labor ha ido más allá y en la actualidad destaca el repositorio digital 
creado por el grupo GHEMAT Brasil, que pone a disposición de la comunidad investigadora fuentes primarias, tales como textos escolares o exámenes de matemáticas, e igualmente actas de eventos y trabajos de investigación actuales, entre otros. En el marco de este ambicioso proyecto conjunto se busca hallar respuesta a las cuestiones relacionadas con la transformación de los saberes y las relativas a los saberes profesionales en tanto que articulación entre saberes de enseñanza y saberes para la docencia. Las Humanidades Digitales se presentan como un ámbito para esa búsqueda de respuestas.

Finalmente, los profesores Carrillo, Moreno y Sánchez-Jiménez nos informan acerca del Centro de Estudios sobre la Memoria Educativa de la Universidad de Murcia (CEME) y de la labor desarrollada en él durante una década de existencia y, en particular, en torno a la Historia de la Educación Matemática. La creación y la existencia misma del CEME ha estado vinculada al interés por la memoria y el patrimonio histórico-educativo en España y la creación de asociaciones, centros de estudio, museos y publicaciones relacionadas con el tema. En este trabajo se informa sobre la actividad llevada a cabo por del CEME, que comprende actuaciones de diferente tipo: exposiciones, publicaciones, organización de eventos, como conferencias y congresos, actividades docentes y, por supuesto, investigaciones por parte de sus miembros. Su función abarca la conservación y la divulgación del patrimonio histórico educativo, así como la investigación y la formación de graduados y posgraduados y, especialmente, el fomento de la investigación histórico educativa.

Las colecciones del CEME (libros, cuadernos, fotografías, tarjetas postales, catálogos de material, materiales didácticos, archivos personales de profesores, grabaciones con testimonios de docentes, etc.), una vez catalogados los materiales, proporciona a los investigadores y estudiosos fuentes para su trabajo y, en ocasiones, motivan la realización de exposiciones temporales, algunas realizadas en el marco de proyectos de investigación, y que suelen contar con el apoyo de otras instituciones y organismos. Actividad que, junto con el desarrollo del Museo Virtual de Historia de la Educación (MUVHE), se inscribe en el museísmo pedagógico y contribuye a la función de divulgación del patrimonio material e inmaterial en historia de la educación. Los autores destacan la relación del CEME con la investigación en historia de las disciplinas, 
especialmente el análisis de las culturas escolares, la evolución de las disciplinas escolares y su código disciplinar, en particular, en historia de la educación matemática. Comentan los paradigmas de investigación en los que se basan las investigaciones en este campo llevadas a cabo por investigadoras del CEME, inscritas en el llamado «Programa epistemológico de investigación en didáctica de la matemática», y describen diferentes clases de actividades realizadas en este sentido y el papel de los recursos aportados por el CEME para la realización de estos trabajos, haciendo un recorrido por los tipos de fuentes y la manera en que estas contribuyen al conocimiento de la intrahistoria de la educación matemática. 